\section{Immune checkpoint inhibitors in combination with radiother- apy as salvage treatment for relapsed/refractory classical Hodgkin lymphoma: A retrospective analysis in 12 patients}

\author{
Elisa Lucchini, ${ }^{1}$ Chiara Rusconi, ${ }^{2}$ \\ Mario Levis, ${ }^{3}$ Francesca Ricci, ${ }^{4}$ \\ and highly active bridge treatment to trans- \\ plant consolidation.
} Armando Santoro, ${ }^{4}$ Umberto Ricardi, ${ }^{3}$ Stefano Volpetti, ${ }^{5}$ Fabio Matrone, ${ }^{6}$ Anna di Russo, ${ }^{7}$ Manuela Caizzi, ${ }^{1}$ Anna Schiattarella, ${ }^{8}$ Francesco Zaja ${ }^{1-9}$

${ }^{1}$ Department of Hematology, Azienda Sanitaria Universitaria GiulianoIsontina, Trieste; ${ }^{2}$ Division of Hematology and Stem Cell Transplantation Fondazione IRCCS Istituto Nazionale dei Tumori, Milano; 3Department of Oncology, University of Torino; ${ }^{4}$ Department of Oncology and Hematology, Humanitas Clinical and Research Center - IRCCS, Rozzano, Milano; ${ }^{5}$ Department of Hematology, Azienda Sanitaria Universitaria Friuli Centrale, Udine; ${ }^{6}$ Department of Radiation Oncology, Centro di Riferimento Oncologico di Aviano (CRO) IRCCS, Aviano, Pordenone; ${ }^{7}$ Division of Radiotherapy Oncology Fondazione IRCCS Istituto Nazionale dei Tumori, Milano; ${ }^{8}$ Department of Radiotherapy, Azienda Sanitaria Universitaria Giuliano-Isontina, Trieste; ${ }^{9}$ Department of Medical, Surgical and Health Sciences, University of Trieste, Italy

\section{Abstract}

The rate of complete remission (CR) with the anti-PD1 immune checkpoint inhibitors (ICI) nivolumab $(\mathrm{N})$ and pembrolizumab (P) in patients with relapsed/refractory $(\mathrm{R} / \mathrm{R})$ classical Hodgkin lymphoma (cHL) is low (20-30\%), and the majority of patients eventually relapse. One strategy to improve their outcome is to combine ICI with radiotherapy (ICI-RT), taking advantage of a supposed synergistic effect. We retrospectively collected data of 12 adult patients with $\mathrm{R} / \mathrm{R}$ cHL treated with ICI-RT delivered during or within 8 weeks from the start or after the end of ICI. Median age at ICI-RT was 37 years, 50\% had previously received an autologous stem cell transplantation (SCT) and 92\% brentuximab vedotin. RT was given concurrently, before or after
ICI in 4, 1 and 7 patients. Median RT dose was $30 \mathrm{~Gy}$, for a median duration of 22 days. Median number of ICI administrations was 15. Overall response and $\mathrm{CR}$ rate were $100 \%$ and $58 \%$. Nine patients received subsequent SCT consolidation ( 7 allogeneic and 2 autologous). After a median follow-up of 18 months, $92 \%$ of patients were in CR. No major concerns about safety were reported. ICI-RT combination appears to be a feasible

\section{Introduction}

Before the era of immunotherapy, the prognosis of patients with relapsed/refractory $(R / R)$ classical Hodgkin Lymphoma (cHL) who fail salvage therapy and autologous stem cell transplantation (SCT) consolidation was poor with an estimated median overall survival of 2 years. ${ }^{1,2}$ In the last few years, the introduction of Brentuximab Vedotin (BV) and immune checkpoint inhibitors (ICI) has offered new therapeutic opportunities for patients with $\mathrm{R} / \mathrm{R} \mathrm{cHL}$.

$\mathrm{BV}$ single agent results in $75 \%$ overall response rate (ORR) in the $\mathrm{R} / \mathrm{R}$ setting, but most patients require further treatment within 1 year. $^{3}$ The ICI Nivolumab (N) and Pembrolizumab $(\mathrm{P})$ bind to $\mathrm{PD}-1$ protein expressed by $\mathrm{T}$ cells, thus inhibiting the binding with PD1-ligand (PD-L1). PD-L1 is overexpressed on the surface of Hodgkin's Reed-Sternberg cells, and the binding with PD-1 inactivates T-cell mediated immune response: this constitutes one of the main mechanisms of tumor escape from immune surveillance. ${ }^{4}$ Treatment with $\mathrm{N}$ and $\mathrm{P}$, which reactivates the immune response against tumor cells, led to nearly $70 \%$ ORR in heavily pretreated cHL patients; ${ }^{5,6}$ however the rate of complete response (CR) is only $20 \%$ with $\mathrm{N}$ and $28 \%$ with P, with a median progression-free survival (PFS) of 14 months. Strategies should therefore be implemented in order to improve the response rates to ICI and to choose the best timing and modality to consolidate the response. Radiotherapy (RT) is an effective therapeutic option in $\mathrm{cHL}$ as a single agent even in the setting of $\mathrm{R} / \mathrm{R}$ disease. ${ }^{7,8}$ Recently, the combination of RT and ICI, given either concurrently or sequentially, has shown to significantly improve the outcome in solid tumors (mainly lung cancer and melanoma).9,10 This synergistic effect still needs to be demonstrated in cHL patients; in fact the activity of RT, the optimal dose, timing of administration and its safety profile when combined with these new agents is not yet defined and only few
Correspondence: Francesco Zaja, Department of Medical, Surgical and Health Sciences, University of Trieste, SC Ematologia Ospedale Maggiore, Piazza dell'Ospitale 1, 34125 Trieste (TS), Italy.

E-mail: francesco.zaja@asugi.sanita.fvg.it

Key words: Nivolumab; pembrolizumab; radiotherapy; Hodgkin lymphoma; ICI-RT.

Conflict of interest: CR: advisory board MSD (2017 and 2018). All the other authors have no conflict of interest to declare.

Contributions: FZ and UR designed the study; EL and ML wrote the manuscript. All the authors contributed to data collection and data analysis, critically revised the manuscript and approved the final version of the paper.

Funding: None.

Availability of data and materials: Data and materials available within the text.

Received for publication: 23 January 2021. Accepted for publication: 30 April 2021

This work is licensed under a Creative Commons Attribution-NonCommercial 4.0 International License (CC BY-NC 4.0).

CCopyright: the Author(s), 2021

Licensee PAGEPress, Italy

Hematology Reports 2021; 13:9080

doi:10.4081/hr.2021.9080

case reports have been published so far. ${ }^{11-13}$ In this report we describe the clinical characteristics and the outcomes of a group of R/R cHL patients who were treated with ICI in combination with RT.

\section{Materials and Methods}

\section{Patients}

In this observational study we retrospectively analyzed the medical records of adult patients with a pathologically confirmed diagnosis of cHL who received ICI-RT at 6 Italian Centers between July 2015 and February 2020. The study was approved by the local Ethical Committee and patients gave written informed consent. The study was registered at Clinicaltrials.gov NCT04419441.

\section{Treatment}

\section{Immune checkpoint inhibitors}

$\mathrm{N}$ and $\mathrm{P}$ were administered at standard dosage as compassionate use or in label indications according to the period of administration. 
CTCAE (common terminology criteria for adverse events) version 4.0.

Patients were treated according to the techniques available at the referring center, which respected the more recently published guidelines. ${ }^{7,14,15}$ Both 3-dimensional conformal RT or intensity-modulated RT solutions were adopted.

Combination treatment was arbitrarily defined when one of these 3 possibilities was met: a) ICI and RT administered concurrently; b) RT administered within 8 weeks before the start of ICI; c) RT administered within 8 weeks after the end of treatment with ICI.

\section{Outcomes}

Response were evaluated according to Lugano Criteria and Lyric Criteria. ${ }^{16,17}$ Adverse events were graded according to

\section{Patients}

Twelve patients were analyzed. Baseline characteristics and previous treatments before ICI-RT are summarized in Table 1.

All patients had a diagnosis of cHL, with initial presentation as advanced GHSG risk group in $58 \%$. The median age of the patients at ICI-RT treatment was 37 years, nobody had a bulky disease, $67 \%$ had a stage I-II disease. Confirmatory tumor biopsy was performed before ICI-RT in 6

\section{Results}

Table 1. Clinical characteristics and previous lines of treatment of 12 patients who were included in the analysis before receiving the combination of ICI and RT.

\begin{tabular}{|c|c|}
\hline Number of patients & 12 \\
\hline Male & 7 \\
\hline Median age at treatment with $\mathrm{N}$ or $\mathrm{P}$, yrs (range) & $37(21-71)$ \\
\hline $\begin{array}{l}\text { Histology at diagnosis } \\
\text { Nodular sclerosis } \\
\text { Mixed cellularity } \\
\text { Lymphocyte depletion } \\
\text { Classical HL, not otherwise specified }\end{array}$ & $\begin{array}{l}6 \\
2 \\
1 \\
3\end{array}$ \\
\hline $\begin{array}{l}\text { GHSG risk at diagnosis: } \\
\text { Advanced } \\
\text { Early favorable } \\
\text { Early unfavorable }\end{array}$ & $\begin{array}{l}7(58 \%) \\
0(0 \%) \\
5(42 \%)\end{array}$ \\
\hline $\begin{array}{l}\text { Stage before treatment with ICI and RT: } \\
\text { I-II } \\
\text { III-IV } \\
\text { Bulky }\end{array}$ & $\begin{array}{l}8(67 \%) \\
4(33 \%) \\
0\end{array}$ \\
\hline $\begin{array}{l}\text { Previous treatment } \\
\text { First-line therapy }(\mathrm{n}=12) \\
\text { ABVD } \\
\text { ABVD/BEACOPP } \\
\text { BV }\end{array}$ & $\begin{array}{c}10 \\
1 \\
1\end{array}$ \\
\hline $\begin{array}{l}\text { Second-line }(\mathrm{n}=11) \\
\text { IGEV + autologous SCT } \\
\text { IGEV } \\
\text { DHAP } \\
\text { ESHAP } \\
\text { DHAOX }\end{array}$ & $\begin{array}{l}4 \\
4 \\
1 \\
1 \\
1\end{array}$ \\
\hline $\begin{array}{l}\text { Third-line }(\mathrm{n}=10) \\
\text { BV } \\
\text { BV + autologous SCT } \\
\text { BV + bendamustine } \\
\text { BV + bendamustine + autologous SCT }\end{array}$ & $\begin{array}{l}4 \\
1 \\
1 \\
1\end{array}$ \\
\hline $\begin{array}{l}\text { ICE } 1 \\
\text { BEACOPP } \\
\text { DHAP }\end{array}$ & $\begin{array}{l}1 \\
1\end{array}$ \\
\hline $\begin{array}{l}\text { Fourth-line }(\mathrm{n}=7) \\
\text { Bendamustine } \\
\text { BV } \\
\text { BV + bendamustine } \\
\text { IGEV } \\
\end{array}$ & $\begin{array}{l}3 \\
2 \\
1 \\
1\end{array}$ \\
\hline
\end{tabular}

BV: Brentuximab Vedotin; SCT: stem cell transplantation. N: Nivolumab; P: Pembrolizumab.

patients (50\%). Most patients $(83.3 \%)$ had received $\mathrm{ABVD}$ as first-line therapy, $58.3 \%$ being primary refractory. All patients were heavily pre-treated, since 7 (58\%) previously received four or more lines of therapy, 6 (50\%) autologous SCT and $11(91.6 \%) \mathrm{BV}$. Only half of the patients received autologous SCT, because the remaining had a chemoresistant disease which never allowed to perform the procedure. Response to the last treatment before ICI-RT was: refractory disease in 6, PR in 2, relapse after transient CR in 4 (median duration of CR: 9 months, range 3-15). The majority of patients $(83 \%)$ received ICI-RT as the fourth or subsequent line of treatment, including 3 patients who received it as the sixth line of therapy and 1 as the seventh line of therapy.

\section{Treatment}

$\mathrm{N}$ and $\mathrm{P}$ were administered at standard dose in $9(75 \%)$ and 3 patients $(25 \%)$, respectively, starting after a median of 3 months (range 0-15) from the end of the last line of treatment. The reason why either $\mathrm{N}$ or $\mathrm{P}$ was chosen was related only to drug availability (compassionate use before registration) and label indication. Median treatment duration and number of administrations of $\mathrm{N}$ and $\mathrm{P}$ were 7 (range 4-19) months and 15 (range 8-32), respectively.

None of the patients had previously received RT in the same sites that were subsequently irradiated together with ICI. Median RT dose was 30 Gy (range 30-40 Gy). RT was delivered in conventional fractionation (1.8-2-0 Gy/die) to all patients for a median duration of 22 days. Doses and sites of irradiation are reported in Table 2.

RT was given concurrently, before or after ICI in 4, 1 (N started 38 days after the end of RT), and 7 patients (RT started after a median of 23 days, range $11-43$, after the last administration of $\mathrm{N}$ or $\mathrm{P}$ ), respectively.

\section{Outcome}

After ICI-RT, 7 patients (58.3\%) were

Table 2. Sites and dose of RT received by the patients in combination with ICI. RT: radiotherapy.

\begin{tabular}{lc}
\hline Sites of RT & \\
Mediastinal & 5 \\
Laterocervical + supraclavear & 4 \\
Iliac and inguinal & 1 \\
Laterocervical + mediastinal & 1 \\
Mediastinal + axillary + supraclavear & 1 \\
Dose of RT (Gy) & \\
30 & $7(58 \%)$ \\
36 & $4(33 \%)$ \\
40 & $1(8 \%)$
\end{tabular}


in $\mathrm{CR}$ and $5(41.7 \%)$ in PR, with two patients still receiving $\mathrm{P}$ at last follow-up. Response was documented by CT-PET in 8 patients and by $\mathrm{CT}$ scan only in 4 patients. Ten patients $(83 \%)$ interrupted treatment with ICI, 9 of them in order to receive allogeneic (7) or autologous (2) SCT consolidation; 1 patient in CR interrupted ICI treatment according to the center choice.

After a median follow-up of 18 months (range 10-52 months) since the starting of ICI-RT, 11 patients (92\%) were alive in CR; 1 patient who progressed after ICI-RT died because of infectious complication developed after a subsequent haplo-identical SCT (Figure 1).

\section{Safety profile}

Six $(50 \%)$ patients reported one or more adverse events (AE). RT-related grade 1 AEs were seen in 4 patients: odynophagia $(\mathrm{n}=1)$, dysphagia $(\mathrm{n}=1)$, dysgeusia $(\mathrm{n}=1)$ and esophagitis $(\mathrm{n}=1)$; all resolved. One patient developed hypothyroidism, which was considered $\mathrm{N}$ related. Two patients developed a grade 3 interstitial pneumonia. The first patient (sequential $\mathrm{N}$ and RT) developed interstitial pneumonia of unknown origin 8 months after the end of mediastinal RT, 9 months after having stopped $\mathrm{N}$ and four months after allogeneic SCT; he was treated with steroids, antiviral and antimycotic therapy, with resolution. The second patient (concomitant $\mathrm{N}$ and RT), developed pneumonia 6 months after the end of mediastinal RT, three months after the end of $\mathrm{N}$ therapy and 1 month after having received autologous SCT. Microbiological analysis from bronchus lavage were negative and lung biopsy showed the pattern of diffuse infiltrative pneumonitis. This patient received steroids and antibiotic therapy, with resolution.

\section{Discussion and Conclusions}

In this retrospective real-life experience in heavily pretreated R/R cHL, ICI-RT led to an ORR and CR of $100 \%$ and $58 \%$, respectively, resulting in a powerful bridge to SCT and allowing 11 out of 12 patients to achieve a long-term CR status.

Only a few reports focused on this topic so far. Qin et al reported 3 patients with $\mathrm{R} / \mathrm{R}$ cHL treated with $\mathrm{N}$ and RT: two patients received RT followed 2 and 3 months later by $\mathrm{N}$, respectively, and one patient received concurrent RT and N. ${ }^{12}$ De Forceville et al reported 2 patients treated with RT in combination with $\mathrm{N}$, in both cases $\mathrm{N}$ was administered within a month after the end of RT. ${ }^{13}$ Quéro et al. reported four patients treated with ICI-RT, ${ }^{11}$ indicating that the combina- tion was feasible and efficacious.

Also, in our cohort the combination was overall well tolerated. The majority of AEs were grade $1-2$, mainly related to radiotherapy, and all resolved. Three patients (25\%) developed AEs who were considered related to ICI: one patient developed a grade 1 hypothyroidism, 5 months after starting N, that was treated with steroids and levothyroxine and two patients $(17 \%)$ developed an interstitial pneumonia, that was considered possibly related to treatment. The AEs did not lead to the interruption of treatment in any case.

Immune-related adverse events are the most remarkable type of toxicity experienced with ICI. Thyroid-related (especially hypothyroidism) are the most frequent, but also immune-mediated rash, hepatitis, pneumonitis, colitis, diabetes, nephritis have been reported. They usually occur at a median of 3 months after starting treatment, and in most cases are mild. ${ }^{5,6}$ The two patients who developed the interstitial pneumonia were heavily pretreated, both received thoracic mediastinal (non-pulmonary) RT, and then developed pneumonia after having received another treatment (1 autologous SCT and 1 allogeneic SCT, respectively): the pathogenesis of these 2 cases of pneumonia might therefore be multifactorial. In the report by Quéro et al., all the four patients treated with ICI in combi-

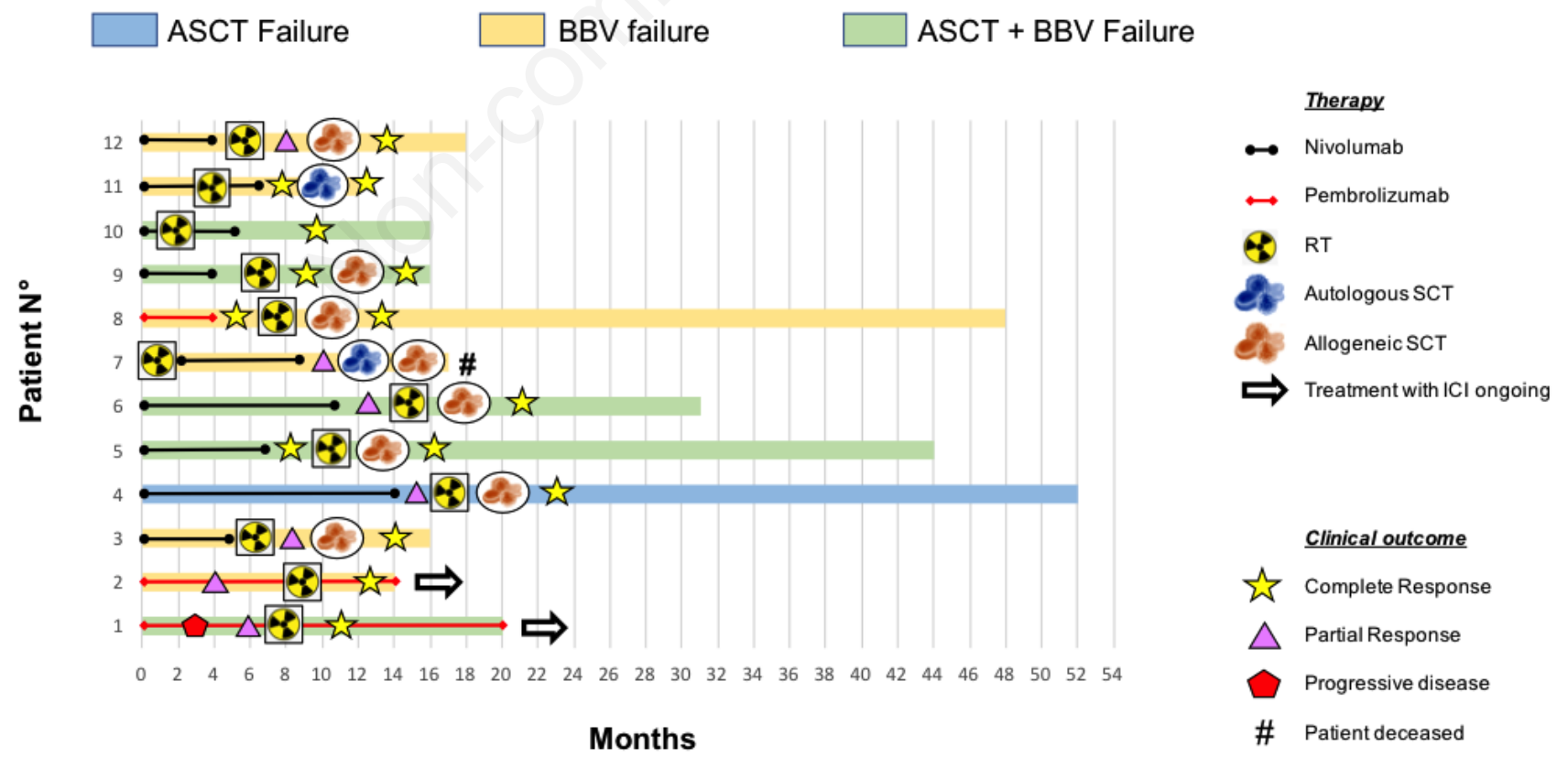

Figure 1. Treatment timing and duration and response onset for the 12 patients enrolled in the study. The color of each bar indicates whether previous autologous SCT and/or brentuximab vedotin (BV) therapy had failed in that patient. The length of the bar shows the observation time since the first day of combined therapy with ICI and RT. Nine patients discontinued ICI, after achieving response, in order to receive stem cell transplant consolidation. Two patients are still continuing ICI after achieving a complete metabolic response with combined ICI-RT. Eleven out of twelve patients are in complete remission at the time of this writing. 
nation with sequential mediastinal RT experienced grade 1-2 lung toxicity; they assumed that it was radiation pneumonitis in two cases, while in the other two cases the etiology was not clarified and probably multifactorial. ${ }^{11}$ Although worthy of further investigations, when combining thoracic RT and ICI therapy, special attention should be paid to the development of interstitial pneumonia.

This retrospective analysis highlights an apparent higher therapeutic activity of this combination (as shown by the clinical case reported in Figure 2) if compared to the historical results with ICI single agent. 5,6

Similar results were already shown in previous reports: the 2 patients described by De Forceville et al had supra-diaphragmatic disease and achieved a CR; ${ }^{13} 3$ patients reported by Quéro et al. ${ }^{11}$ received the combination treatment for supra-diaphragmatic stage II disease; all achieved CR. In the report by Qin et al. ${ }^{12} 2$ out of 3 patients received the combination for a stage II disease (sub-diaphragmatic and supradiaphragmatic respectively), achieving CR. Besides direct cytotoxic effect, it is known that RT may also exerts an immunomodulatory activity, which can be both pro-inflammatory and immunosuppressive, mostly depending on doses and timing of administration. ${ }^{18}$ RT has been shown to promote the release of tumor antigens and to up-regulate MHC-class I molecule expression, thus increasing the immunogenicity of tumor cells. ${ }^{19}$ RT also stimulates the production of pro-inflammatory mediators like ATP and calreticulin, thus promoting the recruitment and activation of antigen-presenting-cells. ${ }^{20}$ On these grounds, the pro-inflammatory, anti-tumour activity of RT can be enhanced by PD-1 blockade, thus producing a possible synergistic effect. ${ }^{21}$ This combination is being investigated also in several solid tumors, showing its efficacy, without raising particular concerns about safety ${ }^{22,23}$

Our study has several limits; the analysis is retrospective and conducted in a limit-

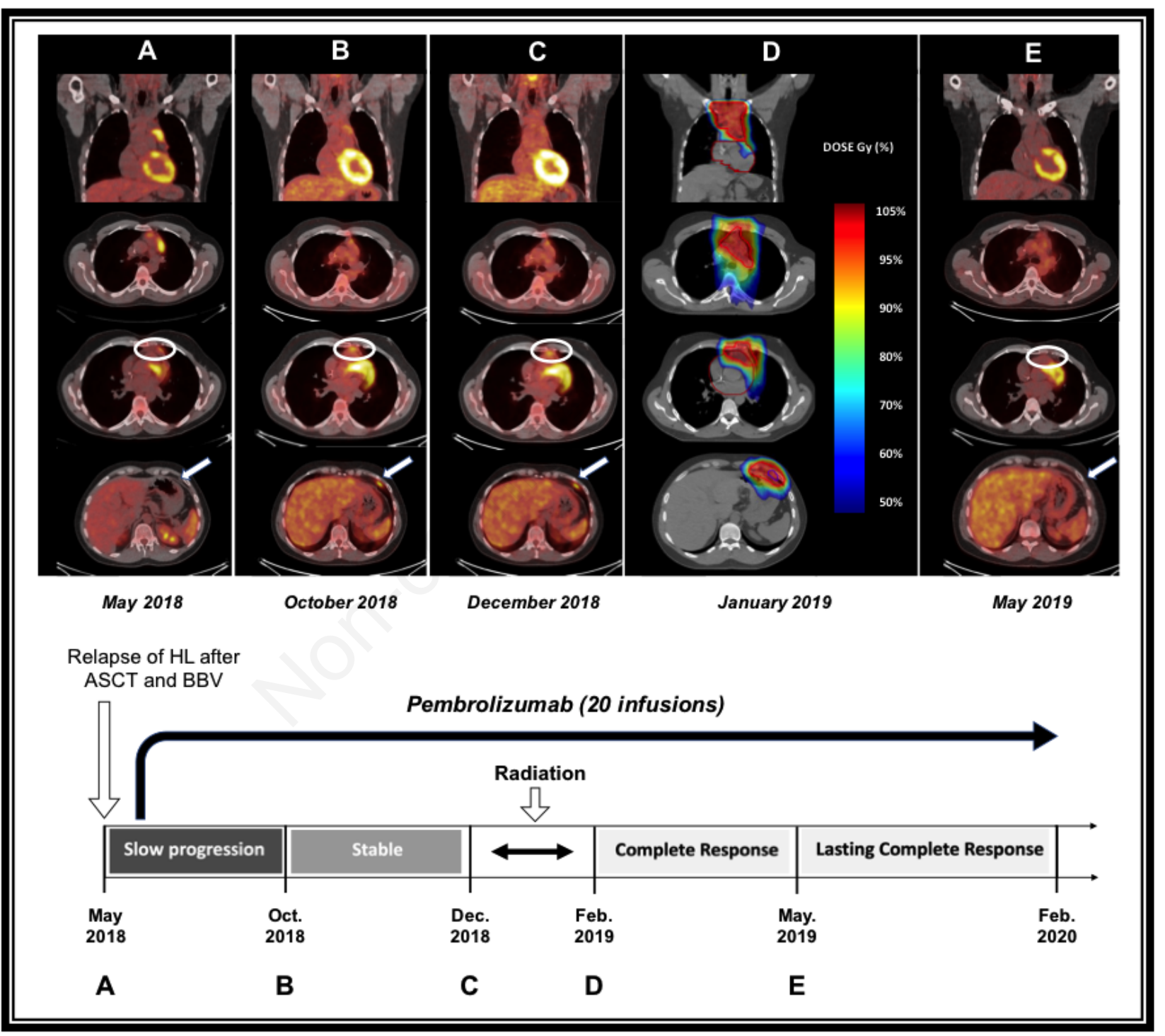

Figure 2. Timeline showing the disease status and therapy in patient 1 of our series. Panel (A) shows disease status before treatment with Pembrolizumab. Panel (B) shows partial response in the upper mediastinum, disease progression in a parasternal lymph node (inside the white circle) and appearance of a new cardiophrenic lymph node (shown by the white arrow) after 4 doses of Pembrolizumab. Panel (C) shows a stable disease after 4 additional doses of Pembrolizumab. Panel (D) shows the dose distribution of the RT treatment. The treatment was planned with an intensity modulated radiotherapy (IMRT) solution with multiple arcs. The prescribed dose was $36 \mathrm{~Gy}$ in 18 fractions. Panel (E) shows the complete metabolic response achieved 3 months after RT. 
ed number of patients; furthermore, the Authors are aware of a possible selection bias due to the fact that other patients that didn't achieve at least a partial response to ICI were probably not considered for RT and were not included into this study. However, the results observed in this cohort of heavily pretreated R/R cHL seem impressive and supports further investigation.

In conclusion, these preliminary results suggest that ICI-RT is safe and effective in $\mathrm{R} / \mathrm{R} \mathrm{cHL}$, and may have a synergistic effect. Further studies are warranted in order to confirm the activity reported in this analysis and better address which might be the best time, sequence and dosing of these two treatments.

\section{References}

1. Majhail NS, Weisdorf DJ, Defor TE, et al. Long-term results of autologous stem cell transplantation for primary refractory or relapsed Hodgkin's lymphoma. Biol Blood Marrow Transplant 2006;12:1065-72.

2. Moskowitz AJ, Perales MA, Kewalramani $\mathrm{T}$, et al. Outcomes for patients who fail high dose chemoradiotherapy and autologous stem cell rescue for relapsed and primary refractory Hodgkin lymphoma. $\mathrm{Br} \mathrm{J}$ Haematol 2009;146:158-63.

3. Younes A, Gopal AK, Smith SE, et al. Results of a pivotal phase II study of brentuximab vedotin for patients with relapsed or refractory Hodgkin's lymphoma. J Clin Oncol 2012;30:2183-9.

4. Keir ME, Butte MJ, Freeman GJ, Sharpe AH. PD-1 and Its Ligands in Tolerance and Immunity. Annu Rev Immunol 2008;26:677-704.

5. Armand P, Engert A, Younes A, et al. Nivolumab for relapsed/refractory classic hodgkin lymphoma after failure of autologous hematopoietic cell transplantation: Extended follow-up of the multicohort single-arm phase II checkmate 205 trial. J Clin Oncol 2018;36:1428-39.

6. Chen R, Zinzani PL, Lee HJ, et al.
Pembrolizumab in relapsed or refractory Hodgkin lymphoma: 2-year followup of KEYNOTE-087. Blood 2019;134:1144-53.

7. Constine LS, Yahalom J, Ng AK, et al. The Role of Radiation Therapy in Patients With Relapsed or Refractory Hodgkin Lymphoma: Guidelines From the International Lymphoma Radiation Oncology Group. Int J Radiat Oncol Biol Phys 2018;100:1100-18

8. Josting A, Nogova L, Franklin J, et al. Salvage radiotherapy in patients with relapsed and refractory Hodgkin's lymphoma: A retrospective analysis from the German Hodgkin Lymphoma Study Group. J Clin Oncol 2005;23:1522-9.

9. Chen L, Douglass J, Kleinberg L, et al. Concurrent Immune Checkpoint Inhibitors and Stereotactic Radiosurgery for Brain Metastases in Non-Small Cell Lung Cancer, Melanoma, and Renal Cell Carcinoma. Int J Radiat Oncol Biol Phys 2018;100:916-25.

10. Antonia SJ, Villegas A, Daniel D, et al. Overall survival with durvalumab after chemoradiotherapy in stage III NSCLC. N Engl J Med 2018;379:2342-50.

11. Quéro L, Gilardin L, Fumagalli I, et al. Anti-PD-1 immunotherapy in combination with sequential involved-site radiotherapy in heavily pretreated refractory Hodgkin lymphoma. Cancer/Radiotherapie 2019;23:132-7.

12. Qin Q, Nan X, Miller T, et al. Complete Local and Abscopal Responses from a Combination of Radiation and Nivolumab in Refractory Hodgkin's Lymphoma. Radiat Res 2018;190:3229.

13. de Forceville L, Deau-Fischer B, Franchi P, et al. Radiotherapy in combination with nivolumab for relapsed/refractory classical Hodgkin lymphoma: About two cases. Cancer/Radiotherapie 2019;23:232-9.

14. Specht L, Yahalom J, Illidge T, et al. Modern radiation therapy for Hodgkin lymphoma: Field and dose guidelines from the international lymphoma radiation oncology group (ILROG). Int J Radiat Oncol Biol Phys 2014;89:854-
62.

15. Filippi AR, Meregalli S, Di Russo A, et al. Fondazione Italiana Linfomi (FIL) expert consensus on the use of intensity-modulated and image-guided radiotherapy for Hodgkin's lymphoma involving the mediastinum. Radiat Oncol 2020;15:62

16. Cheson BD, Fisher R, Barrington SF, et al. Recommendations for initial evaluation, staging, and response assessment of hodgkin and non-hodgkin lymphoma: The lugano classification. J Clin Oncol 2014;32:3059-68.

17. Cheson BD, Ansell S, Schwartz L, et al. Refinement of the Lugano Classification lymphoma response criteria in the era of immunomodulatory therapy. Blood 2016;128:2489-96.

18. Zitvogel L, Kroemer G. Subversion of anticancer immunosurveillance by radiotherapy. Nature Immunol 2015;16:1005-7.

19. Reits EA, Hodge JW, Herberts CA, et al.Radiation modulates the peptide repertoire, enhances MHC class I expression, and induces successful antitumor immunotherapy. J Exp Med 2006;203:1259-71.

20. Gameiro SR, Jammeh ML, Wattenberg $\mathrm{MM}$, et al. Radiation-induced immunogenic modulation of tumor enhances antigen processing and calreticulin exposure, resulting in enhanced T-cell killign. Oncotarget 2014;5:403-16.

21. Sharabi AB, Lim M, DeWeese TL, Drake CG. Radiation and checkpoint blockade immunotherapy: Radiosensitisation and potential mechanisms of synergy. Lancet Oncol 2015; 16:e498-509.

22. Sindoni A, Minutoli F, Ascenti G, Pergolizzi S. Combination of immune checkpoint inhibitors and radiotherapy: Review of the literature. Crit Rev Oncol Hematol 2017;113:63-70.

23. Pitroda SP, Chmura SJ, Weichselbaum RR. Integration of radiotherapy and immunotherapy for treatment of oligometastases. Lancet Oncol 2019;20:e434-42. 